\title{
HIPERTENSÃO ARTERIAL SISTÊMICA E MORBIDADE PSIQUIÁTRICA EM AMBULATÓRIO DE HOSPITAL TERCIÁRIO
}

\author{
ISABELA M. BENSENOR ${ }^{*}$, ALEXANDRE C. PEREIRA**, ANNA C. TANNUR/**, \\ CARLA M. VALERI**, DANIELA AKASHI**, DANIELE Q. FUCCIOLO**, FLÁVIA K. ISSA**, \\ MAURÍCIO L. LOBATO**, SILVIA M. O. TITAN**, TATIANA G. GALVÃO**, PAULO A. LOTUFO***
}

RESUMO - A hipertensão arterial sistêmica representa um dos principais fatores de risco para as doenças cardiovasculares que são a principal causa de morte em nosso meio. Hipertensos que frequentam hospital terciário apresentam elevada co-morbidade incluindo distúrbios psiquiátricos. O objetivo deste estudo é avaliar a morbidade psiquiátrica em hipertensos graves. Este estudo foi realizado em ambulatório geral de hospital terciário: 41 pacientes (26 mulheres e 15 homens) foram submetidos a consulta médica com aplicação do PRIME-MD, um questionário específico para diagnóstico de alterações psiquiátricas a ser realizado pelo clínico. A frequência de distúrbios psiquiátricos diferiu em homens e mulheres: $63,4 \%$ das mulheres na pesquisa apresentavam algum tipo de distúrbio contra 36,6\% dos homens $(\mathrm{p}=0,012)$. A maior parte dos diagnósticos foi de distúrbios do humor representados pela depressão associada ou não a distúrbios ansiosos. A média de idade dos pacientes com distúrbio psiquiátrico foi 47,1 anos contra 59,3 anos dos pacientes sem distúrbio psiquiátrico ( $\mathrm{p}=0,0049)$, mostrando a presença dos distúrbios psiquiátricos em pacientes mais jovens. Outros fatores pesquisados, como a pressão arterial sistólica, a pressão arterial diastólica e índice de massa corpórea não apresentaram diferenças em função dos distúrbios psiquiátricos apresentados. Concluímos que há grande co-morbidade psiquiátrica em hipertensos que frequentam ambulatórios de hospital terciário e que esses distúrbios são mais frequentes em mulheres e em pacientes jovens.

PALAVRAS-CHAVE: hipertensão arterial sistêmica, distúrbio psiquiátrico, depressão, ansiedade, comorbidade.

\section{Hypertension and psychiatric morbidity: a tertiary care setting experience}

ABSTRACT - Arterial hypertension is one of the most important risk factor for cardiovascular disease, the main cause of death in Brazil. Hypertensive patients that have treated in tertiary care hospitals have shown elevated co-morbidity including psychiatric disturbances. Our objective is to study psychiatric co-morbidity among severe hypertensive patients. This study was performed in an out-patient clinic of tertiary medical care setting. Fortyone patients were enrolled in this research ( 26 women, 15 men). They were submitted to a clinical interview and answering the PRIME-MD, a specific questionnaire for diagnosis of psychiatric disturbances (by a general practitioner). Frequencies of psychiatric disturbances were different in men and women: $63.4 \%$ of the women in this study showed some type of psychiatric disturbance versus $36.6 \%$ of men $(\mathrm{p}=0.012)$. The majority of the diagnosis were mood disturbances, mainly depression associated or not with anxious disturbances. Mean age of psychiatric disturbance patients was 47.1 years versus 59.3 years in the patients without psychiatric disturbances $(\mathrm{p}=0.0049)$, showing the presence of psychiatric disturbances in younger patients. Other factors as systolic arterial blood pressure, diastolic arterial blood pressure and body mass index did not show any differences associated with psychiatric disturbance. We conclude that there is a great co-morbidity between high complexity hospitals hypertensive patients and that this type of disturbance is more frequent in women and in younger patients.

KEY WORDS: arterial hypertension, psychiatric disturbance, depression, anxiety, co-morbidity.

*Médico-Assistente do Serviço de Clínica Geral do Hospital das Clínicas (HC) da Faculdade de Medicina da Universidade de São Paulo (FMUSP); **Acadêmicos da FMUSP; ***Professor Assistente-Doutor do Departamento de Clínica Médica da FMUSP. Aceite: 9-março de 1998.

Dra. Isabela M. Benseñor - Av. Dr. Arnaldo, 455 sala 1216 - 01246-903 São Paulo - Brasil. FAX: 0118530992. 
A hipertensão arterial representa um dos principais fatores de risco para as doenças cardiovasculares, que são a principal causa de óbitos em nosso meio ${ }^{1,2}$. A concomitância de distúrbios psiquiátricos, como ansiedade e depressão em hipertensos poderia ter papel na determinação da aderência ao tratamento anti-hipertensivo ${ }^{3}$. A co-morbidade é fato comum ao estudar-se a população que frequenta hospitais terciários. Entre mulheres que procuraram por queixas como cefaléia ou dispepsia, a presença de distúrbio psiquiátrico foi constatada em $90 \%$ dos $\operatorname{cas}^{4}{ }^{4}$. As perspectivas futuras de novo padrão de morbidade e mortalidade indicam que a doença coronária e a depressão serão as principais causas de perda de anos vividos ou da redução dos anos vividos com qualidade ${ }^{5}$.

Como parte inicial de projeto de maior amplitude interessado em estudar as diferenças entre os sexos na determinação dos padrões de mortalidade da sociedade brasileira, realizou-se a aplicação de questionário para identificação de distúrbios psiquiátricos em pacientes hipertensos.

\section{METODOLOGIA}

O estudo foi realizado no Ambulatório Geral e Didático (AGD) do HC-FMUSP no período de setembro1996 a junho-1997, sendo aprovado pelo Comitê de Ética da instituição. Os doentes que participaram assinaram termo de consentimento pós-informação.

Todos os pacientes foram escolhidos aleatoriamente entre os que procuraram atendimento médico com o diagnóstico prévio de hipertensão arterial sistêmica, podendo ou não apresentar outros fatores de risco ou doenças associados. Todos os pacientes foram submetidos a entrevista e consulta médica realizadas por alunos do quinto ano da FMUSP sob supervisão médica direta. Todos os entrevistadores foram treinados anteriormente na aplicação do questionário e na medida da pressão arterial.

O diagnóstico psiquiátrico foi obtido pela aplicação do PRIME-MD ("Primary Care Evaluation of Mental Disorders" $)^{6,7}$ traduzido para o português e adaptado à realidade brasileira pelo setor de Interconsultas do Instituto de Psiquiatria do HC-FMUSP. O PRIME-MD é questionário que operacionaliza os diagnósticos constantes do DSM-IIIR $^{8}$, sendo destinado a sensibilizar o clínico na realização de diagnósticos psiquiátricos. A baixa escolaridade dos pacientes determinou que questionários iniciais fossem lidos pelos entrevistadores para todos os pacientes. Foram incluídos nesta análise somente os pacientes que responderam integralmente o questionário (Anexo) totalizando 41 hipertensos.

A pressão arterial sistêmica (PAS) foi medida e definida de acordo com os critérios do "V-Joint National Comitee on Detection, Evaluation, and Treatment of High Blood Pressure" (JNC-V) , ou seja pressão arterial sistólica (PAS) maior que $140 \mathrm{mmHg}$ e/ou pressão arterial diastólica (PAD) maior que $90 \mathrm{mmHg}$ evidenciaram hipertensão.

O diagnóstico do estado nutricional dos pacientes foi realizado pelo cálculo do índice de massa corpórea (IMC) obtido pela razão do peso em relação ao quadrado da altura. Os indivíduos com IMC superior a 27,3 Kg/ $\mathrm{m}^{2}$ foram definidos como obesos ${ }^{10}$.

A análise estatística foi realizada por análise de variância para as variáveis contínuas e por análise estratificada para as variáveis dicotômicas, com nível de significância menor do que 0,05.

\section{RESULTADOS}

Foram analisados 41 pacientes: 26 mulheres $(63,4 \%)$ e 15 homens $(36,6 \%)$. A média de idade dos pacientes foi $51,2 \pm 13,6$ anos, sendo $50,4 \pm 11,1$ anos para as pacientes do sexo feminino e $52,6 \pm 17,4$ anos para os pacientes do sexo masculino. A média da pressão arterial sistólica foi $167,1 \pm 35,8 \mathrm{mmHg}$ para toda a amostra, sendo $173,3 \pm 37,9 \mathrm{mmHg}$ para as pacientes do sexo feminino e 156,3 $\pm 29,7$ para os do sexo masculino. A média da pressão arterial diastólica foi $103,7 \pm 20,9$ $\mathrm{mmHg}$ para a amostra, sendo $104,1 \pm 22,4 \mathrm{mmHg}$ para o sexo feminino e $103,0 \pm 18,7 \mathrm{mmHg}$ para o sexo masculino.

Foi feito o diagnóstico de presença de distúrbio psiquiátrico em 27 pacientes $(65,8 \%$ da amostra), sendo 19 do sexo feminino (46,3\%) e 8 do sexo masculino (19,5\%), distribuídos da seguinte forma: 24 pacientes com distúrbio do humor $(58,5 \%)$, sendo 19 mulheres $(46,3 \%)$ e 5 homens $(12,2 \%)$, e 3 pacientes com distúrbio do alcoolismo (7,3\%), todos do sexo masculino. Dos pacientes 
Anexo. Adaptação de PRIME-MD utilizado*.

Nome: RG HC:

\section{PRIME-MD}

Questionário do Paciente (assinalar se presente)

No último mês você tem se incomodado muito com:

1. Dor de estômago ( )

2. Dor nas costas, lombar ( )

3. Dor nos braços, pernas ou juntas (joelhos, quadris, etc.) ( )

4. Dores menstruais ou problemas ( )

5. Dor ou problemas durante relação sexual ( )

6. Dores de cabeça ( )

7. Dor torácica ( )

8. Tonturas, vertigens ( )

9. Desmaios ( )

10. Sente seu coração acelerado ou batendo ( )

11. Falta de fôlego ( )

12. Constipação, diarréia ou intestino solto ( )

13. Náusea, gases ou indigestão ( )

14. Sente-se cansada ou com pouca energia ( )

15. Problemas para dormir ( )

16. Sua alimentação está fora de controle ( )

17. Pouco interesse ou prazer em suas atividades ( )

18. Sentindo-se "para baixo", deprimida ou sem esperanças ( )

19. Nervoso, ansioso ou "no limite" ( )

20. Preocupando-se com muitas coisas diferentes ( )

21. Você teve uma crise de ansiedade (sentiu medo súbito ou pânico) ( )

22. Você tem pensado que deveria diminuir a quantidade de álcool que bebe ( )

23. Alguém queixou-se quando você bebeu ( )

24. Você sentiu culpa por causa da bebida ( )

25. Houve um único dia em que você bebeu 5 ou mais doses de cerveja, vinho ou liquor ( )

Globalmente você diria que sua saúde é:

Excelente ( )

Muito boa ( )

Boa ( )

Regular ( )

Ruim ( )

*Instituto de Psiquiatria do HC-FMUSP.

com distúrbio depressivo, 53,6\% apresentavam depressão maior e 4,8\% apresentavam depressão menor. Catorze pacientes apresentavam distúrbios mistos, ou seja, associação de distúrbio ansioso ao distúrbio de humor $(34,1 \%)$ sendo 11 do sexo feminino $(26,8 \%)$ e 3 do sexo masculino $(7,3 \%)$. Os casos de distúrbio ansioso associado distribuíram-se entre distúrbio ansioso sem outra especificação (12,2\%), ansiedade global generalizada (12,2\%) e transtorno do pânico $(9,8 \%)$. Houve predomínio de diagnósticos psiquiátricos entre as mulheres $\left(\chi^{2}=6,19 ; \mathrm{p}=0,012\right)$ em comparação aos homens (Tabela 1).

Entre os pacientes que não apresentavam qualquer tipo de distúrbio psiquiátrico a média de idade foi 59,3 $\pm 13,3$ anos, sendo 53,9 $\pm 10,3$ anos para o sexo feminino e $64,7 \pm 14,4$ anos para o sexo masculino. Entre os pacientes que apresentavam algum tipo de distúrbio psiquiátrico a média de 
Tabela 1. Presença de distúrbio psiquiátrico por sexo obtido pelo PRIME-MD nos pacientes hipertensos atendidos no estudo.

\begin{tabular}{lccc}
\hline & Feminino & Masculino & Total (\%) \\
\hline Sem diagnóstico & 7 & 7 & $14(34,1)$ \\
Transtorno do humor & 19 & 5 & $24(58,5)$ \\
$\quad$ Simples & 8 & 2 & $10(24,4)$ \\
$\quad$ Misto & 11 & 3 & $14(34,1)$ \\
Transtorno do álcool & 0 & 3 & $3(7,3)$ \\
Total & $26(63,4)^{*}$ & $15(36,6)^{*}$ & $41(100)$ \\
\hline
\end{tabular}

*Diferença de diagnóstico psiquiátrico por sexo $(\mathrm{p}=0,012)$.

idade foi $47,1 \pm 11,9$ anos, sendo $49,1 \pm 11,4$ anos para o sexo feminino e $42,2 \pm 12,6$ anos para o sexo masculino. A média de idade foi diferente nos pacientes com e sem distúrbio psiquiátrico, sendo menor nos pacientes que apresentam algum tipo de distúrbio ( $\mathrm{p}=0,0049)$ (Tabela 2$)$. Isso ocorreu às custas dos pacientes com distúrbio do humor que apresentavam média de idade de 47,2 $\pm 11,4$ anos, sendo 49,1 111,4 anos para o sexo feminino e 39,8 $\pm 8,4$ anos para o sexo masculino. A média de idade dos pacientes com distúrbio de humor diferiu da média de idade do restante da amostra $(\mathrm{p}=0,022)$.

Os pacientes com transtorno do alcoolismo apresentavam média de PAS de 153,3 $\pm 5,7 \mathrm{mmHg}$; os com distúrbio do humor, média de PAS de 166,3 $\pm 28,9 \mathrm{mmHg}$, sendo 172,5 $\pm 4,3 \mathrm{mmHg}$ para o sexo feminino e 142,6 $\pm 11,7 \mathrm{mmHg}$ para o sexo masculino. Não houve diferença entre os valores de PAS apresentados de acordo com o tipo de distúrbio psiquiátrico $(\mathrm{p}=0,59)$. Os pacientes com transtorno do alcoolismo apresentavam média de PAD de 110,0 $\pm 6,0 \mathrm{mmHg}$; os com distúrbio do humor média de PAD de 103,6 $\pm 22,8 \mathrm{mmHg}$, sendo $103,9 \pm 25,5 \mathrm{mmHg}$ para o sexo feminino e $102,4 \pm 7,6 \mathrm{mmHg}$ para o sexo masculino. Não houve diferença entre os valores de PAD apresentados de acordo com o tipo de distúrbio psiquiátrico $(\mathrm{p}=0,79)$.

O IMC médio da amostra estudada foi $30,6 \pm 6,3 \mathrm{Kg} / \mathrm{m}^{2}$, sendo $30,11 \pm 6,31 \mathrm{Kg} / \mathrm{m}^{2}$ para o sexo feminino e $31,4 \pm 6,5 \mathrm{Kg} / \mathrm{m}^{2}$ para o sexo masculino Os pacientes sem nenhum distúrbio psiquiátrico apresentavam IMC de $32,1 \pm 6,3 \mathrm{Kg} / \mathrm{m}^{2}$, sendo $30,6 \pm 3,8 \mathrm{Kg} / \mathrm{m}^{2}$ para o sexo feminino e $33,8 \pm 8,1 \mathrm{Kg}$ / $\mathrm{m}^{2}$ para o sexo masculino. Os pacientes com distúrbio psiquiátrico apresentavam IMC médio de $29,8 \pm 6,3 \mathrm{Kg} / \mathrm{m}^{2}$, sendo $30,02 \pm 7,1 \mathrm{Kg} / \mathrm{m}^{2}$ para o sexo feminino e $29,2 \pm 4,1 \mathrm{Kg} / \mathrm{m}^{2}$ para o sexo masculino. O IMC foi $30,1 \pm 6,4 \mathrm{Kg} / \mathrm{m}^{2}$ nos pacientes com distúrbio do humor, sendo $30,0 \pm 7,1 \mathrm{Kg} /$ $\mathrm{m}^{2}$ nos do sexo feminino e $30,1 \pm 3,2 \mathrm{Kg} / \mathrm{m}^{2}$ nos pacientes com distúrbio do humor do sexo masculino. O IMC foi de $27,7 \pm 5,8 \mathrm{Kg} / \mathrm{m}^{2}$ nos pacientes com distúrbio do alcoolismo. Não houve diferença significativa na análise do IMC de acordo com o tipo de distúrbio psiquiátrico. Os pacientes com distúrbio do alcoolismo apresentavam IMC menor, mas o dado não foi significativo ( $\mathrm{p}=0,43$ ) (Tabela 3).

Todos os pacientes da amostra estavam em tratamento para hipertensão arterial sistêmica, fazendo uso de medicação.

Tabela 2. Média de idade por distúrbio psiquiátrico e por sexo obtido pelo PRIMEMD nos pacientes hipertensos atendidos no estudo.

\begin{tabular}{lllc}
\hline & Feminino & Masculino & Total \\
\hline Sem distúrbio & $53,9 \pm 10,3$ & $64,7 \pm 14,4$ & $59,3 \pm 13,3^{*}$ \\
Com distúrbio & $49,1 \pm 11,4$ & $42,2 \pm 12,6$ & $47,1 \pm 11,9^{*}$ \\
\hline
\end{tabular}

*Diferença na média de idade em pacientes com e sem distúrbio psiquiátrico ( $\mathrm{p}=0,0049)$. 
Tabela 3. Índice de Massa Corpórea (IMC) por distúrbio psiquiátrico e por sexo obtido pelo PRIME-MD nos pacientes hipertensos atendidos no estudo.

\begin{tabular}{lcc}
\hline & $\mathrm{IMC}\left(\mathrm{Kg} / \mathrm{m}^{2}\right)$ mulheres & $\mathrm{IMC}\left(\mathrm{Kg} / \mathrm{m}^{2}\right)$ homens \\
\hline Sem distúrbio & $30,6 \pm 3,8$ & $33,8 \pm 8,1$ \\
Distúrbios do humor & $30,0 \pm 7,1$ & $30,1 \pm 3,2$ \\
$\quad$ Simples & $33,2 \pm 8,2$ & $27,6 \pm 4,2$ \\
$\quad$ Misto & $27,7 \pm 5,4$ & $32,0 \pm 2,7$ \\
Distúrbios do álcool & - & $27,7 \pm 5,8$ \\
Total & $30,11 \pm 6,30$ & $31,38 \pm 6,50$ \\
\hline
\end{tabular}

Não houve diferença significativa no IMC por distúrbio psiquiátrico.

\section{DISCUSSÃO}

O questionário PRIME-MD ${ }^{6,7}$ foi desenvolvido para sensibilizar o clínico geral para o diagnóstico de condições psiquiátricas. O clínico sensibilizado poderia fazer uma série de diagnósticos psiquiátricos, inclusive iniciando o tratamento, com encaminhamento dos casos mais complicados a serviços de referência. O PRIME-MD trabalha com a avaliação das doenças mentais mais comuns na população que são as alterações do humor, os distúrbios ansiosos, os distúrbios somatoformes, os transtornos relacionados ao álcool e os transtornos alimentares.

A hipertensão arterial é fator de risco com alta prevalência na população em geral. Na amostra de pacientes hipertensos estudada, houve alta frequência de distúrbios psiquiátricos, totalizando $65,8 \%$. Esta alta frequência de distúrbios psiquiátricos sugere que toda a atenção deva ser dada pelo clínico à avaliação psiquiátrica desses pacientes. Além da óbvia melhoria da qualidade de vida, o controle do distúrbio psiquiátrico poderá ser um fator a mais a garantir a aderência ao tratamento anti-hipertensivo. A transposição desses dados para a população em geral deve ser feita de forma cuidadosa, pois trata-se de amostra de hipertensos que frequenta hospital terciário, sendo casos de maior complexidade com maior freqüência de comprometimento de órgãos-alvo (em relação à hipertensão) e de co-morbidade associada.

Seria o elevado número de distúrbios psiquiátricos apresentados consequência da gravidade dos pacientes estudados, todos portadores de múltiplas patologias? Estudo recente realizado em nosso Serviço com mulheres hipertensas leves e controles normais mostrou também elevada prevalência de sintomas psiquiátricos, utilizando-se também o PRIME-MD ${ }^{4}$. Nessa amostra, as pacientes não apresentavam nenhuma patologia grave, podendo-se concluir que o elevado número de diagnósticos psiquiátricos não seja consequência da gravidade clínica dos casos e sim de uma característica da população que procura um hospital com complexidade terciária. Uma contraargumentação possível é que poderíamos estar utilizando um instrumento diagnóstico (PRIME$\mathrm{MD}$ ) extremamente sensível, o que poderia aumentar os número de diagnósticos feitos. No entanto no artigo original do PRIME-MD, mostrou-se que o questionário possui coeficiente de concordância além da chance (Kappa) de 0,71 com uma acurácia de $88 \%$, valores extremamente elevados que comprovam a sua eficácia ${ }^{6}$.

A diferença entre o número de distúrbios psiquiátricos encontrados entre homens e mulheres coloca o sexo feminino em destaque como fator de risco para o aparecimento de distúrbios psiquiátricos, o que já foi observado em muitos estudos do PRIME-MD ${ }^{7,11,12}$.

A maior frequência de distúrbios psiquiátricos em mulheres teria componentes biológicos (neuro-endocrinológicos) e sociológicos (papel desempenhado na sociedade), que também estariam 
presentes na mulher hipertensa. Em algumas fases da vida (menopausa, divórcio), a mulher estaria mais vulnerável ao aparecimento de distúbios do humor (depressão) ${ }^{7}$. O alto número de diagnósticos encontrados nas mulheres seria, talvez, mais fruto do sexo do que consequência da presença associada da hipertensão.

$\mathrm{Na}$ amostra estudada, os pacientes mais idosos relataram frequência menor de distúrbios psiquiátricos. Os pacientes com algum tipo de distúrbio psiquiátrico apresentavam média de idade inferior à média de idade apresentada pelos pacientes sem distúrbio psiquiátrico. Isso poderia ser consequência de alterações de memória, como já mostrado em outros estudos ${ }^{13}$.

Em relação ao IMC podemos concluir que a média da amostra é extremamente elevada, estando a maioria dos pacientes na faixa da obesidade propriamente dita (acima de $27,3 \mathrm{Kg} / \mathrm{m}^{2}$ ). O IMC é parte fundamental do exame clínico do paciente hipertenso. Neste estudo, tentou-se analisar se haveria alguma alteração do IMC em pacientes hipertensos em função dos distúrbios psiquiátricos associados. Embora tenha-se detectado diferença entre os valores médios do IMC (os pacientes sem distúrbio psiquiátrico apresentando valores de $32,1 \mathrm{Kg} / \mathrm{m}^{2}$ contra somente $29,8 \mathrm{Kg} / \mathrm{m}^{2}$ dos pacientes com distúrbio psiquiátrico), esta não foi significativa. Isso pode ser consequência do pequeno tamanho da amostra. A ausência de transtornos alimentares na amostra pode-se dever a falta de sensibilidade do PRIME-MD no diagnóstico desses transtornos.

Se houve contribuição da medicação anti-hipertensiva sobre o número de distúrbios do humor diagnosticados, é dúvida que o estudo não consegue detectar devido ao seu delineamento transversal. Além disso, por se tratar de hipertensos graves, todos estão em uso de medicação hipotensora.

Concluindo, encontramos alta frequência de distúrbios psiquiátricos em pacientes hipertensos. A maior parte dos distúrbios foi diagnosticada em pacientes do sexo feminino e de faixas etárias inferiores, que representariam os indivíduos de maior risco. Torna-se necessária a capacitação do clínico geral no diagnóstico dos distúrbios psiquiátricos.

Agradecimentos - Aos Drs. Renério Fráguas e Sérgio Henrique Gonçalves do Grupo de Interconsulta do Instituto de Psiquiatria do HC/FMUSP pelo apoio na aplicação do PRIME-MD e à Sra. África Isabel Neuman pelo apoio logístico na execução do estudo.

\section{REFERÊNCIAS}

1. Lotufo PA, Benseñor IJM. Why is stroke mortality higher than CHD in Brazil? In IV Conference on Preventive Cardiology. Montreal, 1997.

2. Lotufo PA. A mortalidade precoce por doenças crônicas nas capitais de áreas metropolitanas do Brasil. Tese (Doutorado), Faculdade de Saúde Pública, Universidade de São Paulo. São Paulo, 1996.

3. Meyer D, Leventhal H, Guttmann M. Common-sense models of illness: the sample of hypertension. Health Psychol 1985;4:115-135

4. Benseñor IJM. Comportamento da pressão arterial na cefaléia crônica diária. Tese (Doutorado), Faculdade de Medicina da Universidade de São Paulo. São Paulo, 1997.

5. Murray CJL, Lopez AD. Alternative projections of mortality and disability by cause 1990-2020: global burden of disease study. Lancet 1997;349:1498-1504.

6. Spitzer RL, Williams JBW, Kroenke K, et al. Utility of a new procedure for diagnosing mental disorders in primary care. JAMA 1994;272:1749-1757.

7. Linzer M, Spitzer R, Kroenke K, et al. Gender, quality of life and mental disorders in primary care: results of the PRIMEMD 1000 study. Am J Med 1996;101:526-533.

8. American Psychiatric Association. Diagnostic and statistical manual of mental disorders. 3.Ed., revised. Washington DC: American Psychiatric Association, 1987.

9. The Fifth Report of the Joint National Committee on Detection, Evaluation, and Treatment of High Blood Pressure (JNC V). Arch Intern Med 1993;153:154-185.

10. Bray GA. An approach to the classification and evaluation of obesity. In Bjoorntop P, Brodoff (eds). Obesity. Philadelphia: JB Lipincott, 1992:294-308.

11. Shumaker SA, Hill DR. Gender differences in social support and physical health. Health Psych 1991;10:80-85.

12. Rapaport MH, Thompson PM, Kelsoe, et al. Gender differences in outpatient research subjects with affective disorders. J Clin Psychiatry 1995;56:67-72.

13. Simon GE, Vonkorff M, Bedirhan U, Gater R, Oye, G, Sartorius N. Is the lifetime of depression actually increasing. J Clin Epidemiol 1995;9:1109-1108. 